\title{
Some influential factors on severity of diabetic foot ulcers and Predisposing of limb amputation: A 7-year study on diabetic patients
}

\author{
Research Article
}

\section{Mostafa Madmoli ${ }^{1}$, Yaghoob Madmoli ${ }^{2}$, Hosein Taqvaeinasab ${ }^{3}$, Mahboobeh Khodadadi ${ }^{4}$, Pouriya Darabiyan ${ }^{4}$, Alireza Rafi ${ }^{5^{*}}$}

\author{
1. Emergency Medical Technician, Dezful University of Medical Sciences, Dezful, Iran \\ 2. MSc Student of nursing, Student Research Committee, \\ Ahvaz Jundishapur University of Medical Sciences, Ahvaz, Iran. \\ 3. Student Research Committee, Dezful University of Medical Sciences, Dezful, Iran \\ 4. Student Research Committee, Ahvaz Jundishapur University of Medical Sciences, Ahvaz, Iran. \\ 5. Student Research Committee, School of Nursing \& Midwifery, \\ Shahid Beheshti University of Medical Sciences, Tehran, Iran.
}

\begin{abstract}
Introduction: Diabetes is the most common metabolic disease and a major global challenge that is the leading cause of death in the industrialized and developing world. Therefore, this study was performed on some influential factors on severity of diabetic foot ulcers and predisposing of limb amputation. Materials and Methods: In this cross-sectional descriptive-analytic study, 4436 cases of diabetic patients admitted to Khatam-ol-Anbia hospital of Shoushtar from 2010 to 2016 were studied. The data in this study included three sections: demographic information, ulcer severity based on Wagner's criterion, and clinical and laboratory data. Then data were analyzed using descriptive and analytical statistical tests. Results: This study included 4436 patients with diabetes with a mean age of $54.36 \pm 42.68$ years. 421 patients (9.4 percent) had a history of diabetic foot ulcers. Also, 385 patients (8.6\%) had a history of limb amputation. In this study, 596 patients $(13.4 \%)$ had a history of smoking and the relationship between smoking and drug use with the severity of ulcer was significant $(p=0.006)$. In this study, in the majority of patients with diabetic foot ulcers $(60.3 \%)$ had severity of ulcer based on Wagner's criterion was second grade. In terms of bacteriology, $57.7 \%$ of the patients during the admission period had cultures of the ulcer site discharge, that the most commonly of mass in $26.7 \%$ of cases was Staphylococcus aureus. In this study, the relationship between type of bacteria and severity of ulcer and limb amputation was significant $(\mathrm{P}<0.05)$, This means that the ulcers that had negative coagulase-staphylococcus bacteria, were higher the severe ulcer and amputations. Conclusion: In this study, the relationship between type of bacteria and severity of ulcer and limb amputation was significant, this means that the ulcers that had negative coagulase-staphylococcus bacteria, were higher the severe ulcer and amputations.
\end{abstract}

Keywords: Diabetic foot ulcer, Limb amputation, Predisposing factors, Risk factor, Staphylococcus aureus.

\section{Introduction}

Diabetes is the most common metabolic disease and a major global challenge that is the leading cause of death in the industrialized and developing world (1-3). This disease is a chronic, metabolic and genetic heterogeneity which is characterized by an increase in blood glucose levels and metabolic abnormalities in carbohydrates, proteins, and fats $(5,4)$. Inappropriate combination (low physical activity and use of unhealthy foods) has increased the uncontrolled prevalence of diabetes in the world (6). In 2014, the

*Corresponding Author:

\section{Alireza Rafi,}

Student Research Committee,

School of Nursing \& Midwifery,

Shahid Beheshti University of Medical Sciences, Tehran, Iran.

E-mail: alirezarafi72@gmail.com global prevalence of diabetes among adults older than 18 years was estimated at $9 \%$. (7). And according to statistics released by the ministry of Health, the prevalence of this disease in Iran is higher than $14 \%$ in the population over the age of 30 , which is mostly in the female population (8). In 2012, diabetes was the main cause of 1.5 million deaths, with $80 \%$ of deaths occurring in low-income and middle-income countries (9). Increased blood glucose can cause chronic and severe complications of diabetes. Complications of diabetes are very common among patients (10). For example, one of the complications of diabetes among diabetic patients is depression, which is one of the most common psychiatric disorders (11). One of the most common and debilitating problems for young people is depression, and so widespread that among psychiatric disorders it is referred to as a mental common cold. $(12,13)$. Depression and occupational stress daily may 
be some health disorders spiritual, mental and physical cause (14). Diabetes mellitus is also one of the most common endocrine complications in thalassemic patients (15). Thalassemia syndrome is a hereditary blood disease. In general, the annual incidence of thalassemic individuals is estimated to be around one hundred thousand worldwide (16-20). Another complication of diabetes is leg-related complications which is for people with diabetes with a variety of disciplines and severe neuropathic, musculoskeletal, and circulatory disorders (21). The risk of having a foot lesions in diabetic patient with that is high (22). These foot ulcers in diabetic patients, constantly infected and has potential for cellulite development and if not treated quickly and appropriately, it leads to blood infection, gangrene, and sometimes leads to amputation (23). Therefore, it is imperative that preventive measures be taken to prevent the diabetic foot ulcer, that involves identifying individuals with risk factors for foot ulcers, teaching the patient and his fellows in the field of foot care and proper and all-round treatment of foot ulcer, which can reduce the amount of amputation more than 50 percent (24).

Because some previous studies have shown that the knowledge of diabetic patients is very weak in relation to the disease, so that in the javadi et al study, $58.3 \%$ had poor knowledge (25). And also due to the importance of examining diabetic foot ulcers and because the results of Vaezi et al. showed that with the increase in age, the rate of follow-up measures for diabetic foot has decreased (24). Considering that, planning to increase the level of health of patients with diabetic foot complications requires knowledge of the risk factors and factors affecting the severity of this ulcer, these risk factors can also lead to limb amputation, Therefore, this study was conducted on files of 4436 diabetic patients for 7 years with the aim of determining Some influential factors on severity of diabetic foot ulcers and predisposing of limb amputation.

\section{Materials and methods}

In this cross-sectional descriptive-analytic study, 4436 cases of diabetic patients admitted to Khatam-olAnbia hospital of Shoushtar city during the period of 7 years from 2010 to 2016 were studied. The patients who were diagnosed with diabetes and had a history of this disease and who were referred to Khatam-ol-Anbia hospital from 2010 to 2016, that were entered in the study. Ten researchers were used to check the records of these patients, that these ten researcher reviewed patients' files by using a researcher-made checklist. In this study, 236 cases were excluded due to incomplete filling or other causes, which were also includes exit criteria.

This article is the result of the research project of Behbahan University of Medical Sciences with the code IR.BHN.REC.1397.9583. After obtaining necessary permits and financial support from Behbahan University of Medical Sciences, this license was referred to the research department of Shoushtar University Of Medical Sciences, and then this research committee issued a permit to Khatam-ol-Anbia hospital, and then files of the patients were used for this study.

The required data for the study were extracted from patients' records from 2010 to 2016 in the medical records section of the hospital. The inclusion criteria included all patients with a medical diagnosis and a history of any type of diabetes, and in each age group and sex. And the exit criteria included other records of patients who had non-diabetic medical diagnosis and also incomplete records. In order to study the records and collect data, was taken an introduction letter from the deputy chancellor of education and research of Shoushtar University of Medical Sciences. Then, the files of patients referring to Khatam-ol-Anbia hospital were used in the archives section. That the required information was collected using a researcher-made checklist from the records. The data in this study included a three-part checklist, the first part containing demographic information that examined gender, age, marital status, occupation, economic status and educational level. The second part, which recorded the type of ulcer grade (diabetic foot ulcer severity), which was present in the patient's case, that this severity of patients' ulcer was according to Wagner's criterion, that is categorized as follows:

Grade zero (skin appears to be healthy), grade 1 (superficial and localized ulcers), grade 2 (deep wound up to the tendon, ligament, bone and joint), grade 3 (deep abscess, osteomyelitis and arthritis), grade 4 (toe gangrene, front part) and grade 5 (gangrene of all parts of the foot). And the third part of the checklist contains clinical and laboratory information of patients that includes predisposing factors and risk factors for diabetic foot ulcer, as well as related to this study and was including BMI, having or not having diabetic foot ulcers, limb amputation, family history of diabetes, having or not having cardiovascular disease, history of drug use, alcohol and smoking, HLP index (including triglycerides, cholesterol, LDL, and HDL), the duration of diabetes, the history of hospitalization due to diabetic foot ulcer or diabetic amputation and the use of antidiabetic drugs. Then, the data were entered into SPSS version 17 , and were analyzed by descriptive statistics including enumerated tables, mean, standard deviation and variance, and analytical tests including $\mathrm{T}$-test, ANOVA, chi-square and chi-square Pearson and at the significant level of $\mathrm{p}<0.05$.

\section{Results}

This study included 4436 patients with diabetes with a mean age of $54.36 \pm 42.68$ years. Of these, 2,489 patients $(56.1 \%)$ were female and the rest were male. The mean BMI in these patients was $33.24 \pm 6.45$, 
Mostafa Madmolil et.al., Some influential factors on severity of diabetic foot ulcers and predisposing of limb amputation

indicating patients with class 1 obesity. And the mean blood glucose level in these patients was $232.43 \pm 23.61$.

421 patients $(9.4 \%)$ had history of diabetic foot ulcers. Also, 385 patients $(8.6 \%)$ had a history of limb amputation. Figure 1

In this study, 596 people (13.4\%) had a history of smoking. Also, 124 people $(2.7 \%)$ had a history of drug use, and $89(2.0 \%)$ had alcohol consumption history. The relationship between smoking and drug use with severity of ulcer was significant $(p=0.006)$, but with alcohol was not significant $(\mathrm{p}=0.09)$. that's mean with drug use and smoking, infection of the foot ulcer increased. Figure 2

In the present study, the severity of diabetic foot ulcers in smokers and non-smokers was different. The majority of smokers $(76.7 \%)$ had grade 3 and nonsmokers $(46.3 \%)$, the severity of their ulcer was second grade. There was a statistically significant relationship between the severity of diabetic foot ulcers with oral hypoglycemic tablets and insulin therapy $(p=0.035)$.

In this study, the relationship between blood glucose level and foot ulcer severity and limb amputation was significant $(\mathrm{P}<0.05)$, This means that people who had more blood sugar, had more severe leg ulcers and more amputations.

In this study, in the majority of patients with diabetic foot ulcers $(60.3 \%)$ had severity of ulcer based on Wagner's criterion was second grade. Also, 10.2\% had grade 1 ulcer, $13.2 \%$ grade 3 ulcer, $8.2 \%$ grade 4 ulcer and $7.7 \%$ had grade 5 ulcers. Figure 3

Also, the relationship between BMI and severity of wound was significant $(p=0.007)$, but with limb amputation was no significant $(\mathrm{p}=0.05)$.

In the present study, the relationship between triglyceride and cholesterol with severity of ulcer and limb amputation was significant $(\mathrm{P}<0.05)$. However, there was no significant difference between LDL and HDL with sever foot ulcer and limb amputation (P> $0.05)$.

Chi-square test showed a significant relationship between economic status and diabetic foot ulcer $(\mathrm{P}=$ 0.009). Also this test showed a significant relationship between education level and limb amputation $(\mathrm{p}=$ 0.001 ), this means that people with lower levels of education were more likely to suffer from limb amputation.

Also, the association between total cholesterol, high density lipoprotein and cholesterol with sex in diabetic patients was significant $(\mathrm{P}=0.003)$.

The mean duration of diabetes was 14.3 years. And $17.2 \%$ of patients over 18 years of age had a history of disease. Also, $20.3 \%$ of subjects mentioned the previous history of hospitalization due to diabetic foot ulcer or diabetic amputation.

Concerning the use of hypoglycemic drugs (Antidiabetic medication), $87.3 \%$ patients used glucose lowering drugs to control their diabetes, diet and insulin injections.
In terms of bacteriology, $57.7 \%$ of the patients during the admission period had cultures of the ulcer site discharge, that the most commonly of mass in $26.7 \%$ of cases was staphylococcus aureus. $21.3 \%$ pseudomonas aeruginosa, $12.3 \%$ non-haemolytic streptococcus, $11.4 \%$ staphylococcus negative coagulase, 8.7\% Escherichia coli, 7.2\% Klebsiella, 6.3\% Enterobacter, $6.1 \%$ Proteus. In general, $79.3 \%$ of the bacteria were gram-positive and the rest were gramnegative.

Chi-square test showed a significant relationship between type of bacteria with severity of ulcer and limb amputation $(\mathrm{P}<0.05)$. This means that the ulcer contains negative coagulase-staphylococcus bacteria, had severity of ulcer and more amputation.

\section{Figure 1: Frequency history of diabetic foot ulcer and limb amputation in diabetic patients}

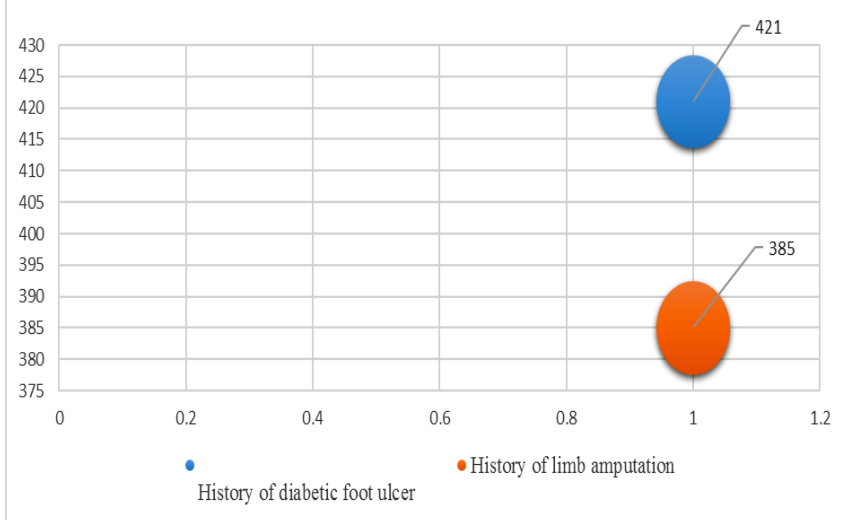

Figure 2: Frequency comparison of history of smoking, drug use and alcohol consumption According to gender

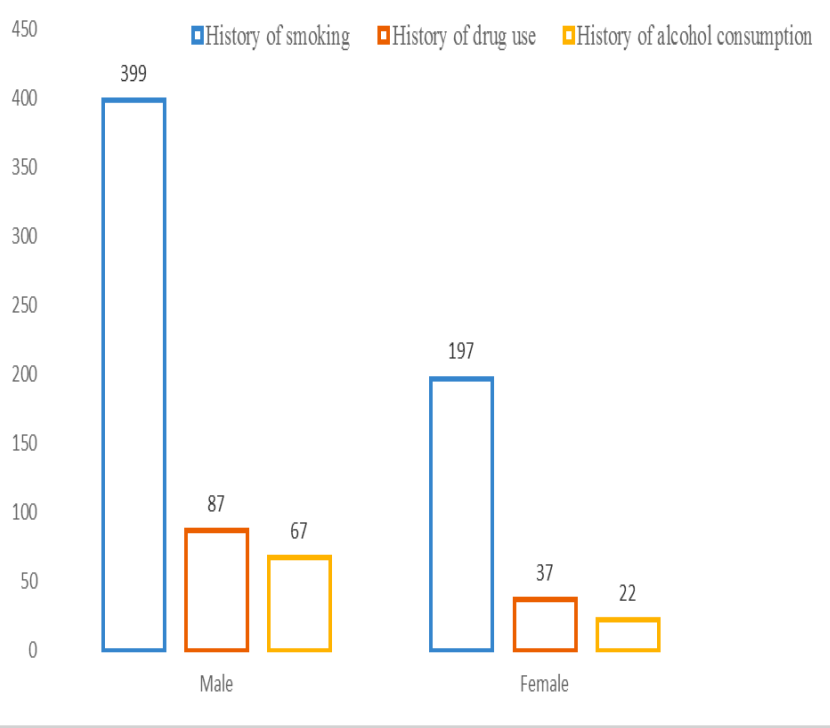


Figure 3: Frequency of ulcer severity based on Wagner ulcer classification in patients with diabetes.

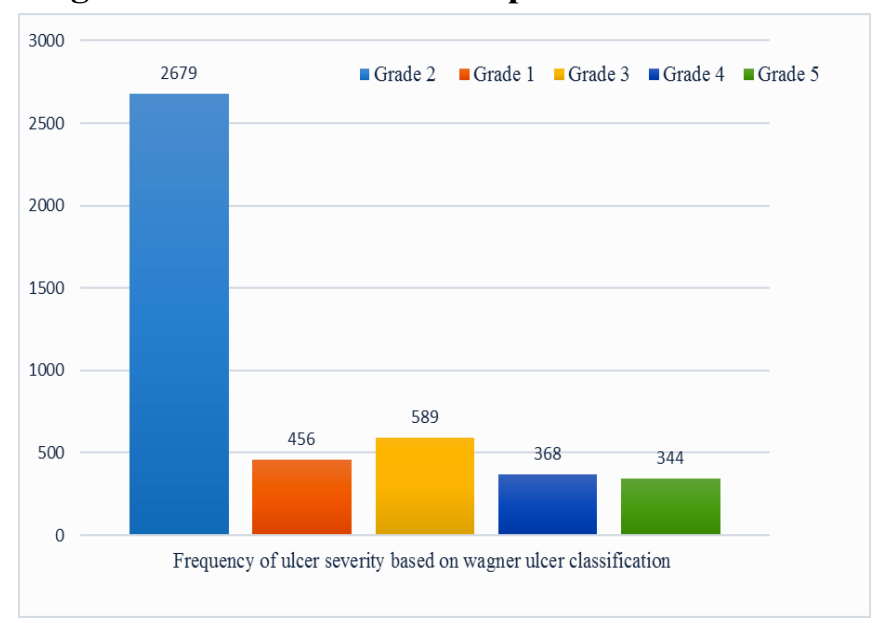

\section{Discussion}

Diabetes is the most common metabolic disease and a major global challenge that is the leading cause of death in the industrialized and developing world (1-3). The risk of having a foot lesions in diabetic patient with is high (22). These foot ulcers in diabetic patients, constantly infected and has potential for cellulite development and if not treated quickly and appropriately, it leads to blood infection, gangrene, and sometimes leads to amputation (23). considering that planning to increase the level of health of patients with diabetic foot complications requires knowledge of the risk factors and factors affecting the severity of this ulcer, these risk factors can also lead to limb amputation, Therefore, this study was performed on files of 4436 diabetic patients for 7 years with the aim of determining Some influential factors on severity of diabetic foot ulcers and predisposing of limb amputation.

In this study, 56.1\% were female and the rest were male, which is in line with the results of studies by urbančič rovan et al (26), madmoli et al (9), and stoekenbroek et al. (27). However, in the study of frykberg et al., $90.3 \%$ of the population were male (28). And in the study of Tan and colleagues, the number of men and women was equal (29). The population of diabetic women in many studies is greater than men, which may be attributed to their gender characteristics, but in terms of complications of diabetes, men develop more than women. In one study, cardiovascular complications, blood lipids, glands and digestive diseases were in women than men and the complications of kidney disease, eye disease, and organ problems in men were higher (30).

In this study, the association between total cholesterol, high density lipoprotein and cholesterol with sex in diabetic patients was significant. The results study of izadi et al (30), also showed that there was no significant relationship between the complications of the disease and the type of treatment, most of the complications related to the disease showed a significant correlation with age, body mass index, triglyceride, total cholesterol, high and low density lipoprotein, so that mean age, body mass index and biochemical parameters were higher in people with complications.

In this study, $9.4 \%$ of the patients had a history of diabetic foot ulcers. Also, $8.6 \%$ had a history of limb amputation. In the study of madmoli et al. (9), the prevalence of lower limb ulcers was $9.1 \%$. In the study of dekker et al., the prevalence of foot ulcer was $7.2 \%$, which is in line with the results of this study (31). However, the prevalence of foot ulcer in this study was higher than other studies. In the study of al-rubeaan et al., The prevalence of foot ulcer was $2.5 \%$ (32). And in the study of stoekenbroek et al., Was $0.34 \%$ (33). Due to the difference in the prevalence of wounds in this study compared to previous studies, it can be attributed to the methods of effective prevention and treatment of foot ulcers, and also the important role of genetics, lifestyle, nutrition and education.

In this study, in the majority of patients with diabetic foot ulcers $(60.3 \%)$ had severity of ulcer based on Wagner's criterion was second grade. Also, 10.2\% had grade 1 ulcer, $13.2 \%$ grade 3 ulcer, $8.2 \%$ grade 4 ulcer and $7.7 \%$ had grade 5 ulcers. In a study by moeini et al. (23), in most patients with diabetic foot ulcers (75\%), their ulcer severity was based on grade 2 Wagner criterion, that was in line with this study. Also, a retrospective cross-sectional study was conducted to determine the frequency of amputation in all patients admitted or outpatient referrals to the shariati center for diabetes clinic due to diabetic foot ulcers to Shariati Hospital in tehran from 2002 to the end of 2011. And the results showed that the average severity of the ulcer was Grade 3 based on Wagner's criterion, also, there was a significant relationship between male sex, previous hospital records, history of amputation, lack of employment, ischemic ulcer, grade 3 ulcer Based on Wagner's criterion with amputation frequency (34). Also, Janmohammadi et al. concluced a study aimed at identifying the predisposing factors of foot ulcer and its treatment methods in diabetic patients, that was on 100 patients with foot ulcer in babol city, and the results showed that there was no significant relationship between sexes with smoking, alcohol and obesity. According to Wagner's classification, the severity of the wound was in most of the referents in Grid 1 and Grade $2(35)$.

In this study, the relationship between blood glucose level and foot ulcer severity and limb amputation was significant, this means that people who had more blood sugar, had more severe leg ulcers and more amputations. In the study of madmoli et al. (9), there was a significant relationship between blood glucose and foot ulcer prevalence. In the study of dekker et al., There was a significant relationship 
between cumulative sugar content and the incidence of diabetic foot disorders (31). The results of a study showed that improved blood glucose control can improve wound healing (36).

The relationship between smoking and drug use with severity of ulcer was significant, but with alcohol was not significant. that's mean with drug use and smoking, infection of the foot ulcer increased. In the present study, the severity of diabetic foot ulcers in smokers and non-smokers was different. The majority of smokers $(76.7 \%)$ had grade 3 and non-smokers $(46.3 \%)$, the severity of their ulcer was second grade. There was a statistically significant relationship between the severity of diabetic foot ulcers with oral hypoglycemic tablets and insulin therapy. shailesh et al. Conducted a study in india aimed at assessing the risk of diabetic foot factors in patients, which was a prospective study in 678 diabetic patients. The results showed that age above 50 years, the length of the disease for 4 years, being rural, treating diabetes for 8 years with insulin, and using tobacco is an important risk factor for the development of diabetic foot ulcers in northern India (37). Also, the results of the study by moeini et al. Showed a significant relationship between the severity of the ulcer and the smoking. The severity of the ulcer in most smokers, grade 3 and non-smokers was grade 2 (23). Also, the study of shailesh et al. (37), has been investigated the relationship between risk factors and severity of ulcer in diabetic foot patients. Which reported a significant relationship between the severity of the ulcer with the smoking and alcohol consumption.

In this study in terms of bacteriology, $57.7 \%$ of the patients during the admission period had cultures of the ulcer site discharge, that the most commonly of mass in $26.7 \%$ of cases was staphylococcus aureus. In the study of bejestani et al., $55.4 \%$ of the patients had cultures of the ulcer site discharge, that in this study too, the most commonly of mass in $26.7 \%$ of cases was staphylococcus aureus (38).

As said, diabetes has multiple risk factors and complications. In a study quoted by mashali et al., was diabetes one of the four major risk factors for cardiovascular disease (39).

\section{Conclusion}

Diabetic foot is associated with an increased risk of death in diabetic patients. The most common cause is neuropathy, leg deformity, leg trauma, upper foot pressure and peripheral vascular disease. That leads to lower limb amputation if not treated properly and with increased mortality, high cost of treatment and reduced quality. In this study, there was a significant relationship between smoking and drug use, blood glucose oral tablet and insulin therapy, triglyceride and cholesterol levels, BS and BMI with severity of ulcer. In this study, the relationship between type of bacteria and severity of ulcer and limb amputation was significant, this means that the ulcers that had negative coagulase-staphylococcus bacteria, were higher the severe ulcer and amputations. Therefore, according to the results of this study, attention to predisposing factors and risk factors of diabetic foot ulcer and severity of ulcers that may cause lower limb amputation, by health care staff members, especially nurses and doctors who care and treat these patients, it seems necessary.

\section{Acknowledgment}

The researchers in this study appreciated and thanked everyone who cooperated with us. The names of the ten researchers who helped in investigating cases and collecting data are as follows:

Mostafa Madmoli, Yaghoob Madmoli, Pouriya Darabiyan, Parsa Madmoli, Mohammad Madmoli, Iman Madmoli, Mohsen Madmoli, Reza Madmoli, Karim Madmoli, Poya Madmoli and Parsa Madmoli.

\section{Conflict of interest}

There are no conflicts of interest in this study.

\section{References}

1. Moslemirad M, Madmoli M, Madmoli Y, Niksefat M. Prevalence of type 1 and type 2 diabetes and its related factors in diabetic patients hospitalized in Khatam-ol-Anbia hospital in Shoushtar, 2014-15: A retrospective study. Journal of Research in Medical and Dental Science. 2018;6(3):421-6

2. Mostafa Madmoli, Zahra Kord, Azita Bandani, Negin Sedighi, Mahla Rezaei Shandiz, Pouriya Darabiyan, Alieh AfsharNia. Epidemiological and clinical study of patients with Alzheimer's in Five Cities of Khuzestan Province in 2016-2018. Medical Science, 2019; 23(95), 1-5

3. Mostafa Madmoli, Mehdi Fallah bagher shaidaei, Akram Rohani, Pouriya Darabiyan, Fariba Mobarez. The correlation between alcohol consumption and reducing the age of cancer incidence in patients with this disease. Medical Science, 2019, 23(95), 48-53

4. Madmoli M, Eilami O, Rezaie K, Aliabad MA, Moslemirad M. Diabetes and The Risk of Suffering Cardiovascular Diseases: A Two-Year Retrospective Study International Journal of Ecosystems and Ecology Science (IJEES). 2018 Jun;8(3): 649-56.

5. Mostafa Madmoli, Yasaman Modheji, Alireza Rafi, Rezvan Feyzi, Pouriya Darabiyan, Alieh AfsharNia. Diabetes and its predictive role in the incidence of Alzheimer's disease. Medical Science, 2019; 23(95), 30-34

6. Raisifar Z, Afshar Nia A, Maghamesi Moarrefi H, Madmoli M. Evaluation of Gi Bleeding Prevalence and Its Related Factors in Diabetic Patients Hospitalized in Khatam-ol-Anbia Hospital During 
2015-16: A Retrospective Study. International Journal of Ecosystems and Ecology Science (IJEES). 2018 June; 8 (3): 609-14.

7. Raisifar Z, Afshar Nia A, Madmoli M, Madmoli Y. The Relationship Between Using Insulin and Suffering Alzheimer's Disease in Patients with Diabetes: A Two-Year Study. International Journal of Ecosystems and Ecology Science (IJEES). 2018 June; 8 (3): 623-28.

8. Madmoli M, Eilami O, Rezaie K, Aliabad MA, Moslemirad M. Diabetes and the risk of suffering cardiovascular Diseases: A two-year retrospective study. International Journal of Ecosystems and Ecology Science (IJEES). 2018 Jun;8(3): 649-56.

9. Madmoli M Rostami F, Mirsami Yazdi N, Mosavi A, Baraz Sh. Evaluation of Prevalence of Diabetic Foot Ulcer and Its Related Factors in Diabetic Patients Admitted to Khatam-ol-Anbia Hospital in Shoushtar During 2015-2016: A Retrospective Study. International Journal of Ecosystems and Ecology Science (IJEES). 2018 June; 8 (3): 545-52.

10. Rostami F, Madmoli M, Mirsami Yazdi N, Baraz Sh. Evaluation of The Prevalence of Lower Limb Amputation and Its Related Factors in Diabetic Patients Admitted to Khatam-ol-Anbia Hospital in Shoushtar During The 2015-2016: A Retrospective Study. International Journal of Ecosystems and Ecology Science (IJEES). 2018 June; 8 (3): 553-60.

11. Larijani B, Khoramshahee M, Bandarian F, Akhondzadeh S. Association of depression and diabetes in the doctor Shariati diabetes clinic and Iranian Diabetes Association. Iranian Journal of Diabetes and Metabolism. 2004 May 15;3(1):77-82.

12. Madmoli Y, Madmoli M, Qashqaei nezhad N, Bosak S. Prevalence of depression and associated factors in adolescents of masjedsoleyman. JPEN. 2016; 2(4):31-40.

13. Madmoli M, Madmoli Y, Bigdeli Shamloo MB, Etebari A, Mahmoodi Kouhi A, Azami M. The Relationship Between Depression and Religiousness in Female High School Students of Masjed Soleyman in 2015. Journal of Pediatric Nursing. 2017 Jun 15;3(4):15-22.

14. Madmoli M, Nikpay S. An Investigation of the Relationship between Spiritual Health and Depression, Anxiety, and Stress among Students of ilam University of Medical Sciences. Journal of Research in Medical and Dental Science. 2018 May 17;6(3):294-300.

15. Azami M, Sayehmiri K. Prevalence of diabetes mellitus in Iranian patients with thalassemia major: a systematic review and meta-analysis. Journal of Mazandaran University of Medical Sciences. 2016 Oct 15;26(141):192-204.

16. Madmoli Y, Aslani A, Ahmadi Y, Mousavi M, Mashalchi H, Niksefat M, Madmoli M. Study Habits and Related Factors in Students of Nursing and Midwifery College of Dezful University of Medical Sciences in 2015. Iranian Journal of Nursing Research (IJNR). 2017.

17. Madmoli Y, Akhaghi Dezfuli SM, Beiranvand R, Saberi Pour B, Azami M, Madmoli M. [An epidemiological and clinical survey of patients with $\beta$-thalassemia in dezful in 2015 (Persian)]. Iran J Epidemiol. 2017; 13(2):145-52.

18. Maraghi E, Adavi A, Madmoli Y, HeidariSoureshjani R, Madmoli M. The Effect of Orem Self-Care on Mental Health of Patients with Thalassemia Major. Journal of Clinical Nursing and Midwifery. 2018;4(3).

19. Madmoli M, Madmoli Y, Rahmati P, Adavi A, Yousefi N, Gheisari Z, Abbaszade Aliabad M. Quality of Life and Some Related Factors in Patients with Beta Thalassemia Major in Southwest Iran. Journal of Client-centered Nursing Care (JCCNC. 2017;3(2).

20. Madmoli Y, Akhaghi Dezfuli SM, Adavi A, Maraaghi E, Heidari Soureshjani R, Madmoli M. The Effect of Orem Self-Care on Mental Health of Patients with Thalassemia Major. Journal of Clinical Nursing and Midwifery. $2018 \mathrm{Jul}$ 1;7(2):108-15.

21. Walsh JW, Hoffstad OJ, Sullivan MO, Margolis DJ. Association of diabetic foot ulcer and death in a population $\square$ based cohort from the United Kingdom. Diabetic Medicine. 2016 Nov 1; 33(11):1493-8

22. Aghakhani N, Broomand A, Alinejad V, Torabi M, Nikoonejad A. The effect of education on quality of life in patients with diabetic foot in educational hospital of urmia. The Journal of Urmia Nursing and Midwifery Faculty. 2016;14(4):380-8.

23. Moeini M, Shahriari M, Yousefi H, Esfandiari J, Babaahmadi M. An investigation on the wound severity and its association with predisposing factors in patients with diabetic foot. Journal of Clinical Nursing and Midwifery. 2017;5(4).

24. Vaezi Aa, Afkhami ardakani M, Evaluation of preventive measures of diabetic foot in diabetics. daneshvar medicine journal 2005; 12 (57):61-68

25. Javadi A, Javadi M, Sarvqdi F. Evaluation of knowledge, attitude and practice of diabetic patients referring to Qazvin diabetes mellitus center towards diabetes mellitus. JBUMS. 2005;11(3):46-51.

26. Urbančič Rovan V, Rovan J. An exploration of diabetic foot screening procedures data by a multiple correspondence analysis. Slovenian Journal of Public Health. 2017 Mar 1; 56(1):65-73. doi: 10.1515/sjph-2017-0009

27. Stoekenbroek RM, Lokin JLC, Nielen MM, Stroes ESG. How common are foot problems among individuals with diabetes? Diabetic foot ulcers in the Dutch population. Diabetologia. 2017; 60: 12711275. doi: $10.1007 / \mathrm{s} 00125-017-4274-7$

28. Frykberg RG, Gibbons GW, Walters JL, Wukich 
DK, Milstein FC. A prospective, multicentere, openlabel, single-arm clinical trial for treatment of chronic complex diabetic foot wounds with exposed tendon and/or bone: positive clinical outcomes of viable cryopreserved human placental membrane. Int wound j. 2016; 14: 569-577. doi: 10.1111/ iwj.12649

29. Tan JH, Hong CC, Shen L, Tay EY, Lee JK, Nather A. Costs of Patients Admitted for Diabetic Foot Problems. Ann Acad Med Singapore. 2015 Dec; 44 (12): 567-70.

30. Izadi N, Rahimi M, Rezvanmadani F, Shetabi H, Darbandi M. A survey on epidemiology of type II diabetes in patients referring to the diaabetes clinic in kermanshah province during 2013-14: A short report. 201790-83 (1)16;

31. Dekker RG, Qin C, Ho BS, Kadakia AR. The effect of cumulative glycemic burden on the incidence of diabetic foot disease. J Orthop Surg Res. 2016 Nov18; 11(1): 143. doi: 10.1186/s13018-016-047-y

32. Al-Rubeaan K, Al Derwish M, Ouizi S, Youssef AM, Subhani SN, Ibrahim HM, et al. Diabetic foot complications and their risk factors from a large retrospective cohort study. PLoS One. 2015 May 6; 10(5): e0124446. doi: 10.1371/ journal.pone. 0124446

33. Stoekenbroek RM, Lokin JLC, Nielen MM, Stroes ESG. How common are foot problems among individuals with diabetes? Diabetic foot ulcers in the Dutch population. Diabetologia. 2017; 60: 12711275. doi: 10.1007/s00125-017-4274-7

34. Mashaikhi M, Larijani B, Mohajerani MR. Investigation of frequency of amputations in patients with diabetic foot ulcers hospitalized in Shariati Hospital 1381-1390. Iranian Journal of Diabetes and Lipid Disorders. 2014; 12 (6): 554-43. 35. Janmohammadi N, Moazzezi Z, Ghobadi P, Haddadi R, Montazeri M. Evaluation of the risk factors of diabetic foot ulcer and its treatment in diabetic patients, Babol, North of Iran. Iranian Journal of Endocrinology and Metabolism. 2010; 11(2): 121-5.

36. Vella L, Gatt A, Formosa C. Does Baseline Hemoglobin A1c Level Predict Diabetic Foot Ulcer Outcome or Wound Healing Time. J Am Podiatr Med Assoc. 2017 Jul; 107(4):272-279. doi: 10.7547/15-176.

37. Shailesh K, Shahim A, Shokkum M. prevalance of diabetic foot ulcer and and association risk factors in diabetic patients from north India. The journal of diabetic foot complication the journal of diabetic foot. 2012; 4 (4): 83-91.

38. Shahrad Bejestani H, Motabar AR. Assessment of diabetic foot ulcer's predisposing factors and its outcomes in patients with diabetic foot syndrome hospitalized in Hazrat Rasoul-e-Akram Hospital in Tehran during 1996-2001. Razi Journal of Medical Sciences. 2004 Jun 15;11(39):77-83.

39. Hadi Mashali, Fatemeh Toleideh, Rezvan Rahmani, Pouriya Darabiyan, Mostafa Madmoli. The predictive role of Hyperlipidemia in the incidence of ACS in patients referring to Shahidzadeh Hospital in Behbahan in 2016 -2017. Medical Science, 2018; 22(94), 566-570. 